MATEC Web of Conferences 13,04030 (2014)

DOI: $10.1051 /$ matecconf/ 20141304030

(C) Owned by the authors, published by EDP Sciences, 2014

\title{
Modal Analysis of a Centrifugal Pump Impeller Using Finite Element Method
}

\author{
Muhammad Ashri ${ }^{1, a}$, Saravanan Karuppanan ${ }^{1}$, Santosh Patil ${ }^{1}$ and Idris Ibrahim ${ }^{1}$ \\ ${ }^{1}$ Department of Mechanical Engineering, Universiti Teknologi PETRONAS, Bandar Seri Iskandar, \\ 31750 Tronoh, Perak, Malaysia.
}

\begin{abstract}
The turbo machinery design has evolved rapidly in the last 50 years. The development in the design of any turbo machinery involves interdisciplinary process, namely stress analysis, vibration analysis, fluid dynamics, thermodynamics and the material selection. One of the major and common analyses used in the development of any mechanical part subjected to dynamic loading is the modal analysis. In this paper, the dynamic characteristics of an impeller of a centrifugal pump were studied. The dynamics characteristics studied were limited to the natural frequencies and the mode shapes. The impeller chosen for the study was a single-entry impeller with radial vanes. Finite element method (FEM) was used since it has proven to be an alternative approach to the experimental method for the vibrational behavior analysis of a component or a system. Modeling and simulation of the impeller have been carried out using ANSYS Bladegen and ANSYS Workbench software, respectively. The eigenvalues and the eigenvectors were observed, and they represent the frequencies and the corresponding mode shapes of the impeller, respectively. The results showed that the varying number of impeller blades and impeller blade thickness had minimal effect on frequencies. However, the change to the impeller disk thickness had significant effects on the natural frequency of the impeller.
\end{abstract}

\section{Introduction}

Impellers are rotating piece of equipment inside a pump. Its function is to increase the pressure of liquid, gases and vapour and eventually force the fluid to move to the desired direction. Impellers transfer the energy from the motor to the fluid. The rotating impeller will create centrifugal force and accelerate the fluid outward of the pump. Impellers are usually made from iron, steel, bronze, brass, aluminium or plastic. There are many types of impeller, namely open impeller, semi-open impeller, closed impeller, single-entry impeller and double-entry impeller. The type of impeller chosen is based on the system pressure requirement and the type of fluid transferred by the pump. 
An impeller consists of a rotating disc called the hub, to which blades are attached. The rotating motion of the impeller blades moves the fluid outwards [1]. The impeller is always placed directly onto the shaft of the electric motor so that it spins at a very high speed, which leads to a continuous vibration.

Centrifugal pump is the most common equipment in many industries because it is simple, effective and inexpensive [2]. The performance of the pump is greatly influenced by the impeller. The variant of the pump is often designed by the modification of the impeller. The impeller is often exposed to hostile operating conditions such as impact from the incoming fluid, vibration and fatigue from the continuous rotation of the impeller itself. These harsh operating conditions will affect the performance of the pump. Therefore the dynamic analysis of the impeller is vital for the evaluation of the performance of a pump. One such work was carried out by Ziaei Rad [3], where modal testing and modal analysis of a radial impeller for varying blade and disk thickness showed significant changes to the natural frequencies. Subramaniam [4] carried out a modal analysis of a centrifugal pump impeller to understand the frequency change due to the change in blade thickness. In this paper, the modal analysis was extended further by the inclusion of the blade number and also the disk thickness. The scope of the modal analysis was restricted to the natural frequencies and the mode shapes of the impellers.

\section{Finite Element Modelling of Impeller Blades}

The modal analysis of open type single-entry impellers with varying parameters was carried out using finite element method (FEM). FEM is a widely used method to perform vibrational analysis [3-5]. It is capable to analyze any arbitrary shaped components and produce acceptable results. In this study, the FEM tool chosen for the impeller modal analysis was the ANSYS Workbench software. The 3D models of the impellers for the analysis were generated using the Bladegen feature of the ANSYSWorkbench [6]. The modelling process is a part of the pre-processing stage in finite element analysis. The generated impeller models were then linked to ANSYS modal analysis platform for the continuation of modal analysis. The base impeller geometry considered for the analysis is shown in Figure 1. From this base impeller geometry, several other impeller geometries were designed, each of them with a different blade thickness, blade number and disk thickness. Table 1 shows the different geometries of the impeller.

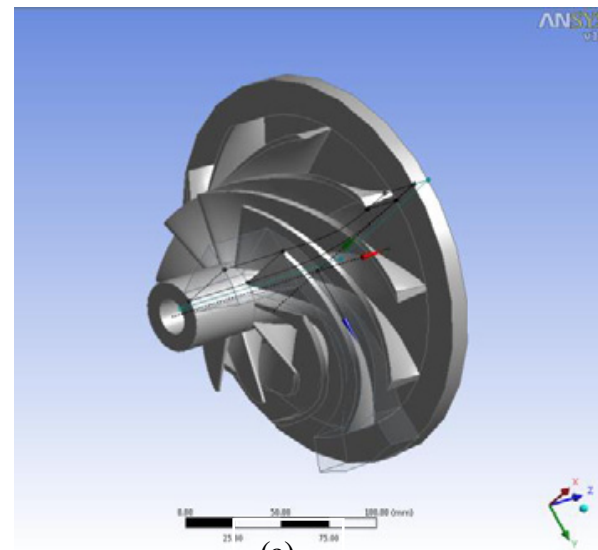

(a)

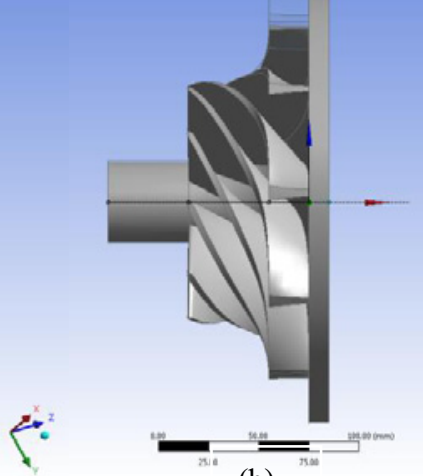

(b)

Figure 1. Base impeller geometry, (a) Isometric view, (b) Side view

Table 1. Pump impeller geometry variation 


\begin{tabular}{|c|c|c|c|}
\hline $\begin{array}{c}\text { Impeller } \\
\text { no }\end{array}$ & Blade thickness & Blade number & Disk thickness \\
\hline Base & $3 \mathrm{~mm}$ & 10 & $25.016 \mathrm{~mm}$ \\
\hline 1 & $2 \mathrm{~mm}$ & Same as base & Same as base \\
\hline 2 & $4 \mathrm{~mm}$ & Same as base & Same as base \\
\hline 3 & Same as base & 5 & Same as base \\
\hline 4 & Same as base & 15 & Same as base \\
\hline 5 & Same as base & Same as base & $12.516 \mathrm{~mm}$ \\
\hline 6 & Same as base & Same as base & $37.516 \mathrm{~mm}$ \\
\hline
\end{tabular}

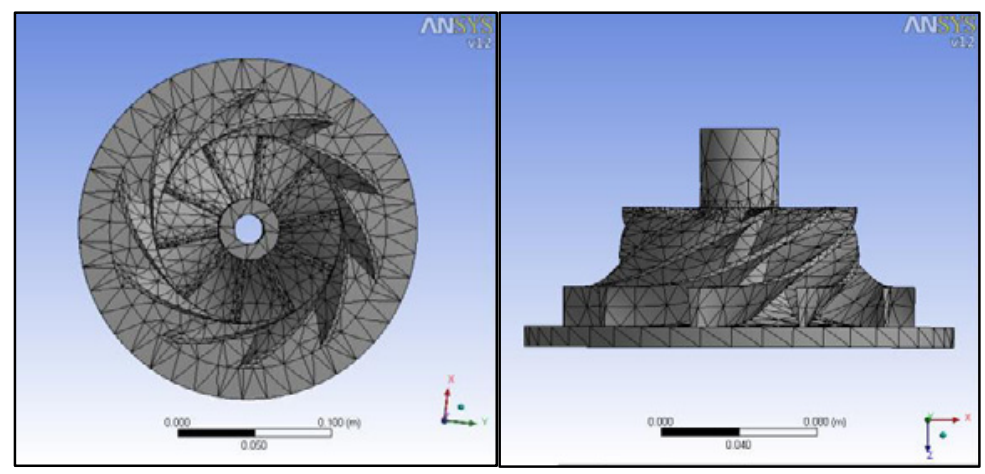

(a)

(b)

Figure 2. Meshed model of base impeller (a) Top view and (b) side view.

The material considered was structural steel. The material properties assigned were density of $7850 \mathrm{~kg} / \mathrm{m}^{3}$, Young's modulus of $200 \mathrm{GPa}$, Poisson's ratio of 0.3 , yield strength of $250 \mathrm{MPa}$ and tensile strength of $460 \mathrm{MPa}$.

The following step was to carry out a proper meshing (discretization) of the models. Meshing can be very time-consuming and error-prone if it was done manually. In recognition of this problem, a large number of methods have been devised to automatically generate the mesh. In ANSYS meshing, mesh settings like surface or edge sizing, meshing method, sphere of influence and more can be set by the user just by picking the geometry. These features are preserved in the model and when the model design is changed, the user just need to "Update Mesh" to get a similar mesh [6]. The meshed model of the base impeller is shown in Figure 2. Similar meshing was carried out for the other impeller designs. The impeller blade models have been meshed using SOLID185. SOLID185 [6] is a 3D element type suitable for computation of natural frequency, deformation, stress and strain with appropriate utilization of time and computer resources.

The inner surface of the impeller hub has been constrained in all degrees of freedom (DOF) prior to modal analysis. The model analysis was then carried out to determine the frequencies and the corresponding mode shapes. In this analysis, the modal frequency was limited to 10 modes. The reason was because the lowest frequencies are the prominent modes which dominate the higher frequencies.

\section{Results and Discussion}

Modal frequency is also known as natural frequency where resonance will occur if the forced frequency matches. Figure 3(a) shows the graph of modal frequency versus impeller blade numbers. The change in frequency at each mode was found to be very small when the impeller blade numbers was changed. This shows that the number of blades is not the main parameter affecting the system's 
natural frequency. Thus they did not give a significant change to the impeller natural frequency [7]. The mass and stiffness of blades are negligible when compared to the impeller mass and stiffness as a whole. Hence, the variation in the number of blades had minimal effect on the natural frequencies. The sample mode shapes of the base impeller (i.e. 10 numbers of blades and blade thickness of 0.3 $\mathrm{mm}$ ) are represented in Figure 3(b). The mode shapes represent the bending modes and deformations of the impeller and impeller blades. It was observed that the mode shapes of the impeller did not change much for different number of blades. The same observation was found for the impellers with different blade thickness and disk thickness.

The results for modal frequency versus impeller blade thickness are shown in Figure 4. It was observed that the modal frequency of the impeller have a slight increasing trend [7]. This result is consistent with the results obtained by Subramaniam [4] who investigated the modal analysis of a centrifugal pump impeller. In both cases, the natural frequency of impeller increased when the blade thickness was increased. This is explained by the stiffness theory of a material. When the mass of the material is increased, the stiffness of the material will also increase. The increase in the natural frequency can be caused by the increase in the stiffness of the structure.

The results for modal frequency versus impeller disk thickness are shown in Figure 5. At the $1^{\text {st }}$ natural mode the difference in frequency for each disk was not too large but the difference became more significant for natural modes 2, 3 and 5. For higher modes, the differences in frequencies for all the 3 impellers become small and less significant. This can be seen in Figure 5 where the $10^{\text {th }}$ natural mode frequency has smaller frequency change when compared to its lower natural frequency modes. The frequency difference between each impeller was due to the mass increment of the impeller. When the disk thickness of the impellers increased, the mass also increased, leading to the increase in the stiffness of the system. Therefore, it can be deduced that the impeller disk thickness is a major parameter in influencing the natural frequency of the impeller.

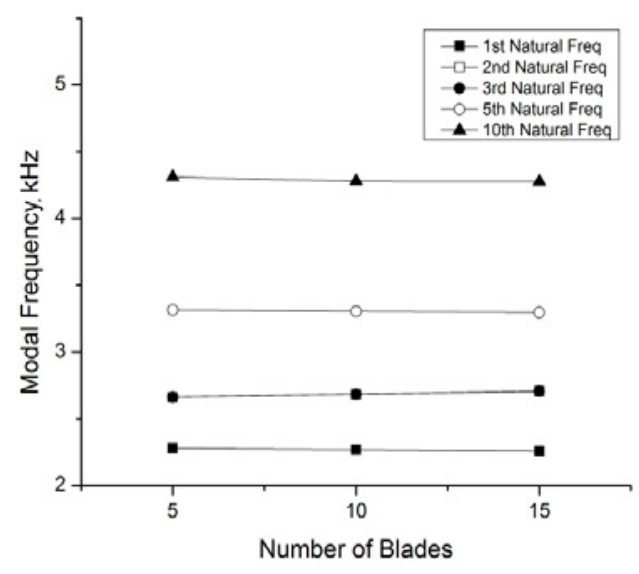

(a)

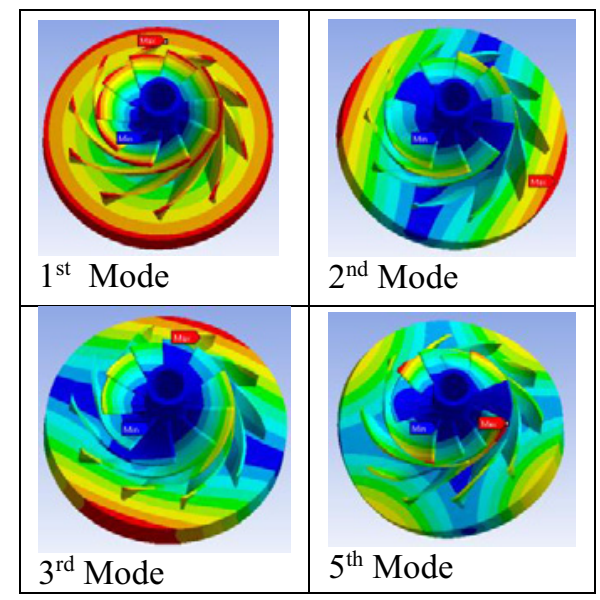

(b)

Figure 3. (a) Frequency versus blade numbers, (b) Sample modes shapes of 10 impeller blades with $t=0.3 \mathrm{~mm}$ (Base Impeller) 


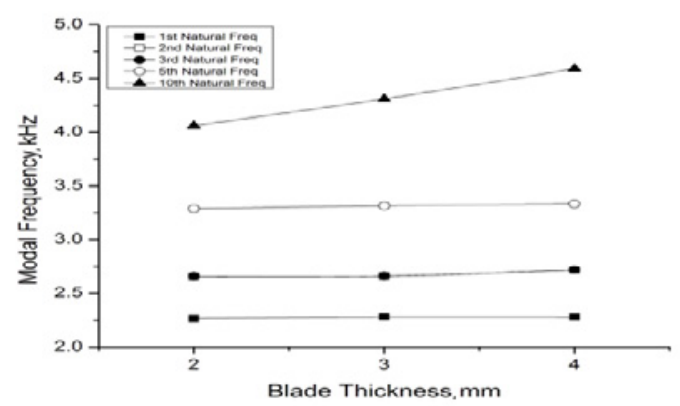

Figure 4. Frequency versus blade thickness

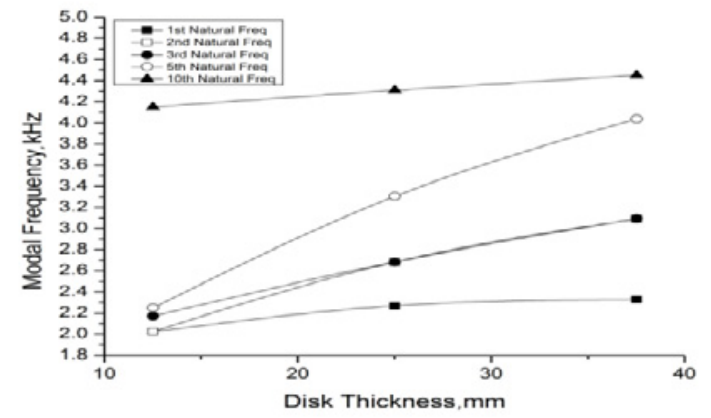

Figure 5. Frequency versus disk thickness

\section{Conclusion}

The modal analysis of the impellers with multiple parameters was carried out by the finite element analysis. The natural frequencies and the mode shapes of the impellers were extracted at different modes. The main conclusions obtained from the modal analysis are summarized as follows;

1. For the analysis of impellers with different number of blades, the modal frequency for each impeller was not significantly different.

2. The natural frequency of the impeller increased slightly as the thickness of the impeller blades increased. This increase in natural frequency can be attributed to the increase in the stiffness of the structure.

3. The impeller disk thickness is a major parameter in influencing the natural frequency of the impeller. The frequency increases with the increase in impeller disk thickness.

\section{References}

1. A.T. Oyelami, S.B. Adejuyigbe, M.A. Waheed, A.K. Ogunkoya, D. Iliya, The Pacific Journal of Science and Technology. 13(1), 24-33 (2012)

2. C. Jacobsen, The Centrifugal Pump. 5th Edition, John Wiley \& Sons, 128 (2010)

3. R. S. Ziaei, Iranian Journal of Science \& Technology, Transaction B - Engineering. 29(B2), 157-169 (2005)

4. L. Subramaniam, European Journal of Scientific Research. 79(1), 5-14 (2012)

5. S. Patil, S. F. Patil, and S. Karuppanan, International Journal of Applied Engineering Research, 8(14), 1685-1694 (2013)

6. ANSYS Release 12.0, Structural Analysis Guide, SAS IP, Inc. (2009)

7. Muhammad Ashri, Modal Analysis of Pump Impeller Using Finite Element Method. Final Year Project, Mechanical Engineering Department, Universiti Teknologi PETRONAS, Bandar Seri Iskandar, Perak (2013) 